\title{
Evaluating the influence of subject-related variables on EMG-based hand gesture classification
}

\author{
Francesco Riillo, Lucia Rita Quitadamo, Francesco \\ Cavrini, Giovanni Saggio \\ Department of Electronic Engineering \\ University of Tor Vergata \\ Rome, Italy \\ Francesco Cavrini, Carlo Alberto Pinto, Nicola \\ Cosimo Pastò \\ Captiks s.r.l. \\ Rome, Italy
}

\author{
Laura Sbernini \\ Department of Experimental Medicine and Surgery \\ University of Tor Vergata \\ Rome, Italy
}

Emanuele Gruppioni

INAIL Centro Protesi

Vigorso di Budrio (Bologna), Italy

\begin{abstract}
In this study we evaluated the effect of subjectrelated variables, i.e. hand dominance, gender and experience in using, on the performances of an EMG-based system for virtual upper limb and prosthesis control. The proposed system consists in a low density EMG sensors arrangement, a purpose-built signal-conditioning electronic circuitry and a software able to classify the gestures and to replicate them via avatars. The classification algorithm was optimized in terms of feature extraction and dimensionality reduction. In its optimal configuration, the system allows to accurately discriminate five different hand gestures (accuracy $=\mathbf{8 8 . 8 5} \pm \mathbf{7 . 1 9 \%}$ ). Statistical analysis demonstrated no significant difference in classification accuracy related to hand-dominance (handedness) and to gender. In addition, maximum accuracy in dominant hand is achieved since first use of the system, whilst accuracy in classifying gestures of the non-dominant hand significantly increases with experience. These results indicate that this system can be potentially used by every trans-radial upper-limb amputee for virtual/real limb control.
\end{abstract}

Keywords-EMG; hand dominance; subject's experience; pattern recognition; amputees.

\section{INTRODUCTION}

In the last decades, surface electromyographic (sEMG) signal acquired on human forearms has been used as input to control a real prosthesis [1] or a virtual device [2], either for interactive or clinical/rehabilitative [3] purposes.

Transradial upper-limb amputees (amputation occurred below elbow) constitute a considerable portion of EMGcontrolled devices users, since the replacement of missing arm functionalities can extensively improve their quality of life. Also the visual-sensorial feedback, following the prosthetic/virtual hand movements, has been recently proposed as a promising methodology to alleviate the phantom limb pain [4-5], an invalidating condition which affects between $50 \%$ and $80 \%$ of amputees [6].

Standard EMG-controlled devices have typically relied on the detection of weak/strong contractions of just two forearm muscles, to perform very simple movements (e.g. hand opening and closing) and this has restricted their usability by amputees [7]. To avoid these limitations, pattern recognition on multiple forearm muscle signals has been proposed to discriminate hand movements [8]. Extracted patterns of EMG activity, specific for each hand movement, allow to increase the amount of information and to realize a more natural, and hence satisfactory, reproduction of the gestures. Fundamentally, a pattern recognition-based system consists of three main steps: 1) EMG signal acquisition by means of an array of sensors; 2) feature extraction, consisting in the calculation of relevant characteristics from the signals, e.g. mean, energy, waveform length, etc. (see [9] for a review); 3) feature translation, or classification, to assign the extracted features to the class (gesture) they most probably belong to. Once the gesture attempted by the user of the system is recognized, it can be mapped towards the controlled device.

In this study we propose a low density sEMG-based system for the recognition of hand gestures to be further replicated via a virtual limb in 3D computer graphics (avatar), useful in rehabilitation of amputees. It implements several different feature extraction modalities and dimensionality reduction techniques. The system was tested with 20 ablebodied subjects, 10 males and 10 females. A comparison of classification accuracy obtained by feeding the classification algorithm with different feature vectors was performed.

In order to improve the performances and the usability of the system, it was necessary to adapt it to the user's peculiarities. For this reason, differences in classification accuracy while performing gestures with the dominant and the non-dominant hand were investigated, together with differences in female and male subjects' performances. This allowed testing its suitability for upper-limb amputees, independently from the amputation site and muscles anatomy. Moreover, we investigated the effect of the experience in using the system, by analyzing separately "inexperienced" (first use) and "trained" (already experienced) subjects. 
By determining the influence of these three variables on classification accuracy, we inferred useful information on how the system could be an efficient solution for the implementation of EMG-piloted devices, either virtual or real, potentially for every upper-limb transradial amputee.

\section{MATERIALS AND METHODS}

\section{A. Subjects}

Testers were twenty able-bodied subjects, ten males and ten females, free of known muscular and/or neurological diseases, $31.85 \pm 10.6 y$ aged. According to their familiarity with the interface, 11 subjects were considered as "inexperienced" and 9 as "trained". Each subject gave informed consent before performing experiments. Also, to determine handedness, each subject was asked for his/her hand preference in most of the daily activities. Eighteen subjects resulted dominant in right hand, 2 in left hand.

\section{B. Setup}

Six commercial active sEMG sensors (Ottobock $13 \mathrm{E} 200=50,27 \times 18 \times 9.5 \mathrm{~mm})$ were placed on the subjects' forearm using a silicone bracelet, as depicted in Fig.1 a-d: sensors were placed equally spaced in the bracelet (Fig. 1a), in such a way that the first sensor was placed on the flexor carpiradialis muscle (Fig. 1b) and the sixth sensor on the brachioradialis muscle (Fig. 1c). The bracelet was placed around the forearm, $5 \mathrm{~cm}$ below the elbow (Fig. 1d). This configuration was chosen to simulate the positioning of the prosthesis sensors on amputees' forearms.

Sensors operated in $0 \div 5 \mathrm{~V}$ range, bandwidth of $90-450 \mathrm{~Hz}$ and Common-Mode Rejection Ratio (CMRR) $>100 \mathrm{~dB}$. Data were collected using a purpose-built acquisition system (12 bits $\mathrm{A} / \mathrm{D}$ converter, $1 \mathrm{kHz}$ sampling frequency).

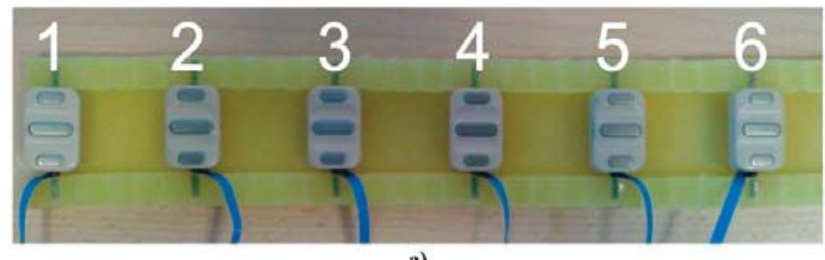

a)

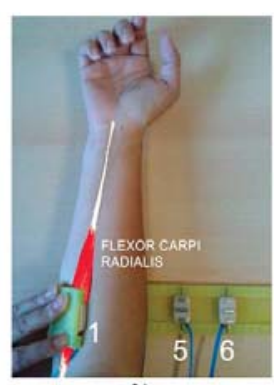

b)

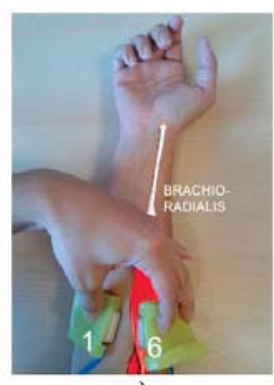

c)

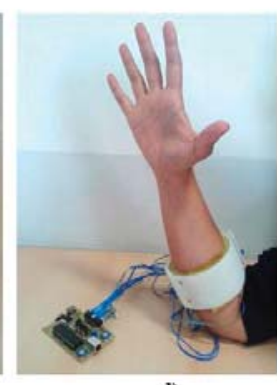

d)
Figure 1: Positioning of the EMG sensors and bracelet. a) the six sensors equally spaced in the bracelet; final bracelet dimensions are $51.3 \times L \times 7 \mathrm{~mm}$ where $\mathrm{L}$ depends on subject's forearm diameter b) sensor 1 positioning; c) sensor 6 positioning; d) bracelet positioning on the forearm.

\section{Experimental procedure}

The subjects were sitting in a comfortable chair in front of a PC monitor, where the following gestures to be performed were depicted (see Fig. 2):

1) Rest: hand relaxed.

2) Fist: hand with all fingers closed.

3) Pinch: hand with thumb and finger touching as if picking a small object.

4) Spread: hand open and stretched.

5) Pointing: hand with all fingers closed with the index pointing.

Every gesture was randomly repeated 10 times and recorded for $2 \mathrm{~s}$. We empirically determined gestures duration by means of preliminary studies. As steady-state sEMG signal are more robust than transient signal for classification purposes [10-11], transitions between gestures were not recorded.

The whole recording procedure was performed twice, once with the dominant hand and once with the non-dominant hand. Half the subjects, randomly selected, started the recording session with the dominant hand and the other half with nondominant hand.

\section{Feature extraction}

After acquisition, raw EMG data were segmented using the overlapped windowing technique [11]: the windows length was fixed to $300 \mathrm{~ms}$, $75 \mathrm{~ms}$ of delay between two successive (overlapped) windows. This timing was chosen in order to fulfill the requirements of real-time applications, such as the control of virtual hands or real prosthesis. For each sensor and each window, features were extracted; in particular, by indicating with $x_{i}$ the $i^{\text {th }}$ time sample in a window and with $N$ the total length of the window (in samples), the following time-domain features were used:

- Mean (M): it is defined in Eq. 1 and represents the mean value of the EMG amplitude.

$$
M=\frac{1}{N} \sum_{i=1}^{N} x_{i}
$$

- Root Mean Square (RMS): it is defined in Eq. 2 and represents the mean power of the signal.

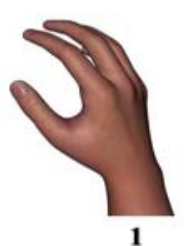

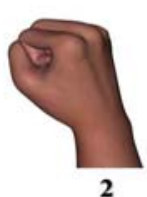

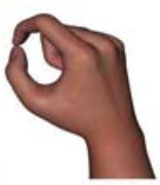

3

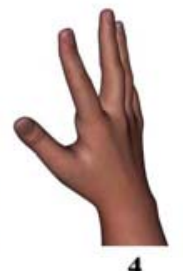

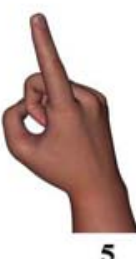

Figure 2: The five hand gestures. 


$$
R M S=\sqrt{\frac{1}{N} \sum_{i=1}^{N} x_{i}^{2}}
$$

- Willison Amplitude (WA): it is defined in Eq. 3 and represents the number of counts for each change in the EMG signal amplitude that exceeds a predefined threshold, set to avoid background noise-induced counts. It is related to the level of muscle contraction.

$$
\begin{aligned}
& W A=\frac{1}{N} \sum_{i=1}^{N-1} f\left(\left|x_{i}-x_{i+1}\right|\right) \\
& f(x)=\left\{\begin{array}{l}
1, x \geq \text { threshold } \\
0, \text { otherwise }
\end{array}\right.
\end{aligned}
$$

The threshold value was set to $1.22 \mathrm{mV}$, as in our preliminary studies with Ottobock $13 \mathrm{E} 200=50$ it was found as the most performing configuration for WA.

- Slope Sign Change (SSC): it is defined in Eq. 4 and represents the number of times the slope of the EMG signal changes sign.

$$
\begin{aligned}
& S S C=\frac{1}{N} \sum_{i=2}^{N-1} f\left[\left(x_{i}-x_{i-1}\right) \times\left(x_{i}-x_{i+1}\right)\right] \\
& f(x)=\left\{\begin{array}{l}
1, x \geq \text { threshold } \\
0, \text { otherwise }
\end{array}\right.
\end{aligned}
$$

The threshold leading to the highest performance was $2.44 \mathrm{mV}$.

Features were tested in single mode and in combination of two, three and four. A total of 15 different configurations were analyzed. After feature extraction, Principal Component Analysis (PCA) was applied to reduce dimensionality of the feature vector $[12,1]$. PCA has been shown to be an effective technique to reduce the data dimensionality in EMG classification problems, obtaining better classification accuracies than algorithms which attempt to determine the best feature subset [13]. The first four principal components (PCs) were retained for the classification phase, as sufficient to achieve a cumulative variance greater than $95 \%$.

\section{E. Classification and statistical analysis}

To compare classification performances achieved with different feature combinations, a 5 folds cross-validation approach was implemented on the entire dataset: the 10 gesture repetitions were randomly divided into 5 subsets of 2 repetitions; 4 subsets were used to calculate PCs and train the classifier and the last one was used to test the classifier. The procedure was repeated until each possible subset was used for testing the classifier.

An Artificial Neural Network (ANN) with 10 neurons in the hidden layer and trained with scaled conjugate gradient back-propagation was implemented. The number of neurons of the hidden layer was empirically determined in previous tests. During training, $15 \%$ of the training dataset was used as a validation dataset in order to improve generalization properties and validate network configuration [14]. The

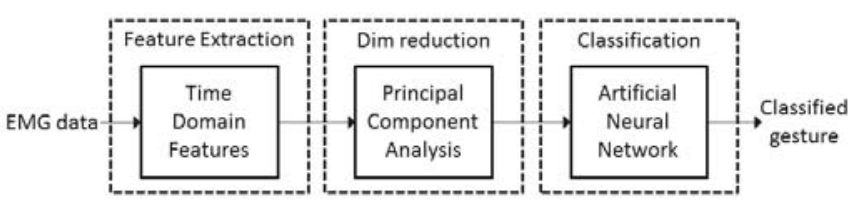

Figure 3: Block diagram of pattern recognition-based system.

implementation of the whole proposed pattern recognition system is shown in Fig.3.

Results obtained by the best feature vector were analyzed by means of paired t-test, in order to detect any statistically significant difference in the accuracies achieved with dominant and non-dominant hand. Then to investigate any statistical effect of the gender (male, female) and of the subjects' experience with the system (inexperienced, trained) on classification accuracy, a two-way ANOVA test was performed on dominant and non-dominant datasets separately. A threshold of 5\% was set for the accepted error.

\section{RESULTS}

\section{A. Selection of the best feature vector}

Table 1 shows the accuracies, averaged over the 20 subjects, and the standard deviation obtained for every different feature vector. Accuracy ranges from $82.93 \pm 9.29 \%$ to $88.85 \pm 7.19 \%$. The highest accuracy was obtained by using a combination of M, RMS and WA as input features. From now on, all the presented results will refer to this optimal configuration.

\section{B. Statistical analysis: handedness, gender and experience effects}

The t-test applied to the accuracies achieved in the classification on the dominant vs. non-dominant hand gestures pointed out no statistically significant differences in their means $(\mathrm{t}=2.25, \mathrm{p}=0.78)$. This indicates that the system was able to discriminate hand gestures with the same accuracy independently from handedness.

Results of two-way ANOVAs, applied separately to dominant and non-dominant hand accuracy, are reported in Table 2. Test on dominant hand discloses no effect of the

TABLE 1: CLASSIFICATION ACCURACY AND STANDARD DEVIATION FOR THE DIFFERENT IMPLEMENTED FEATURE VECTORS.

\begin{tabular}{|c|c|c|}
\hline Features Vector & Accuracy [\%] & S.D. \\
\hline M & 87.79 & 7.48 \\
\hline RMS & 87.65 & 7.8 \\
\hline WA & 83.96 & 9.54 \\
\hline SSC & 82.93 & 9.29 \\
\hline M, RMS & 87.75 & 7.67 \\
\hline M, WA & 88.69 & 6.95 \\
\hline M, SSC & 87.97 & 6.72 \\
\hline RMS, WA & 88.53 & 7.07 \\
\hline RMS, SSC & 87.71 & 6.83 \\
\hline WA, SSC & 84.39 & 9.2 \\
\hline M, RMS, WA & 88.85 & 7.19 \\
\hline M, RMS, SSC & 88.33 & 7.21 \\
\hline M, WA, SSC & 87.94 & 6.73 \\
\hline RMS, WA, SSC & 88.01 & 6.8 \\
\hline M, RMS, WA, SSC & 88.51 & 6.82 \\
\hline
\end{tabular}


TABLE II: ANOVA RESULTS.

\begin{tabular}{|c|c|c|c|c|}
\hline & \multicolumn{2}{|c|}{$\begin{array}{c}\text { Dominant } \\
\text { Hand }\end{array}$} & \multicolumn{2}{c|}{ Non-dominant Hand } \\
\cline { 2 - 5 } & $\mathbf{F}$ & $\mathbf{p}$ & $\mathbf{F}$ & $\mathbf{p}$ \\
\hline Gender & 1.01 & 0.33 & 2.03 & 0.17 \\
\hline Experience & 0.87 & 0.36 & 7.17 & $\mathbf{0 . 0 2}$ \\
\hline $\begin{array}{c}\text { Interaction } \\
\text { (Gender * Experience) }\end{array}$ & 0.86 & 0.37 & 0.1 & 0.76 \\
\hline
\end{tabular}

gender $(\mathrm{F}(1,19)=1.01, \mathrm{p}=0.33)$ and the experience $(\mathrm{F}(1,19)$ $=0.87, \mathrm{p}=0.36)$. Also there is no interaction between the two variables $(F(1,19)=0.87, p=0.36)$. Test on non-dominant hand, instead, shows a statistically significant effect $(\mathrm{F}(1,19)=$ $7.17, \mathrm{p}=0.02$ ) of the experience on classification accuracy.

In Figure 4 the mean and the S.D. relative to classification accuracy in inexperienced and trained subjects, in dominant hand $(88.09 \pm 5.48 \%, 89.69 \pm 10.30 \%)$ and non-dominant hand $(86.82 \pm 5.38 \%, 92.19 \pm 5.13 \%)$, are reported. Note that SD increased in dominant hand accuracy following experience; however no strong evidence $(p>0.05)$ of a real increase could be found. Finally for non-dominant hand accuracy, the gender and the interaction between gender and experience are not statistically significant (respectively $\mathrm{F}(1,19)=2.03, \mathrm{p}=0.17$ and $\mathrm{F}(1,19)=0.1, \mathrm{p}=0.76)$.

\section{DISCUSSION AND CONCLUSIONS}

In this study we investigated the influence of subjectrelated variables (hand dominance, gender and experience in using) on EMG-based hand gesture classification, particularly focusing on devices useful for upper-limb amputees, and we developed strategies for improving system performances.

The proposed system, based on low density sEMG sensors, a combination of M-RMS-WA as feature vector, PCA for dimensionality reduction and classification with ANN, achieved an accuracy of $88.85 \pm 7.19 \%$ in the discrimination of 5 different hand gestures (20 able-bodied subjects). Statistical analysis revealed that classification accuracy and, in

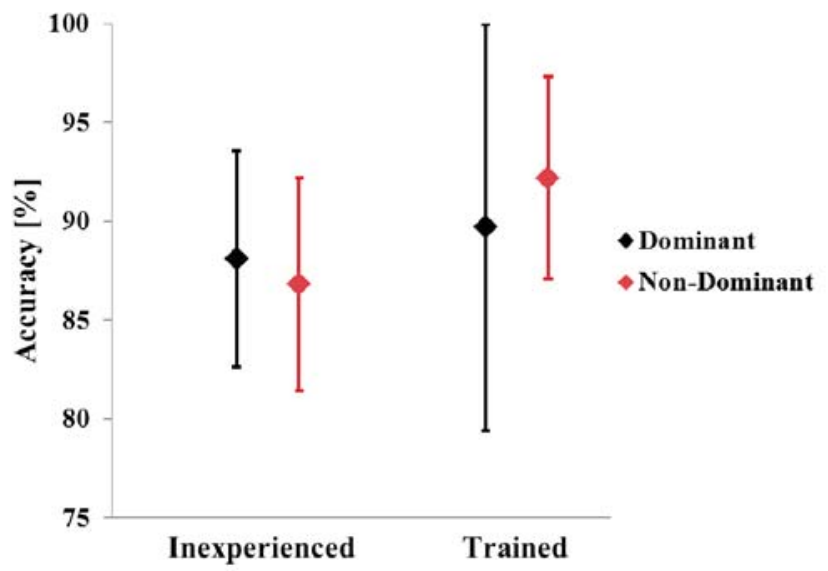

Figure 4: Mean and S.D. of the classification accuracy achieved by inexperienced and trained subjects with dominant and non-dominant hand. Difference in mean values of accuracy in non-dominant hand is statistically significant $(F(1,19)=7.17, p=0.02)$. Difference in dominant hand accuracy is not statistically significant $(F(1,19)=0.87$, $\mathrm{p}=\mathbf{0 . 3 6 )}$. consequence, prosthesis/virtual hand control do not depend on hand dominance (the ability with one hand rather than the other does not affect movements detection performances) nor on user's gender (anatomical differences in musculature between male and female do not affect movement detection performances). This suggested that the proposed system could be potentially used by every amputee subject (female/male, dominant/non-dominant hand amputee) with similar results. In addition, the statistically significant effect of the experience, noticed only in non-dominant hand accuracies, highlighted that non-dominant hand could benefit from extended use of the system. Differently, maximum performance is reached from the very first use of the system with the dominant hand.

Given the encouraging results obtained on able-bodied subjects (high accuracy, same performances both with nondominant hand and different gender), our system will be further tested on amputee subjects. Besides, this work will be extended by investigating the performances of new feature vectors, also in the frequency and time-frequency domains, and new dimensionality reduction algorithms (e.g. Common Spatial Patterns [15-16]), in order to further improve control and usability for amputee subjects.

\section{ACKNOWLEDGMENT}

This study was supported by the "Trattamento della sindrome dolorosa da arto fantasma con tecniche di realtà virtuale" project, by Centro Protesi INAIL and Dipartimento di Ingegneria Elettronica - Università di Tor Vergata, 2010.

\section{REFERENCES}

[1] G.C. Matrone, C. Cipriani, E.L. Secco, G. Magenes, M.C. Carrozza, "Principal components analysis based control of a multi-DoF underactuated prosthetic hand", J Neuroeng Rehabil, vol. 23, pp. 7-16, Apr 2010.

[2] G. Li, A.E. Schultz, T.A. Kuiken, "Quantifying pattern recognitionbased myoelectric control of multifunctional transradial prostheses", IEEE Trans Neural Syst Rehabil Eng, vol. 18, no. 2, pp. 185-192, Apr 2010.

[3] E. Scheme and K. Englehart, "Electromyogram pattern recognition for control of powered upper-limb prostheses: state of the art and challenges for clinical use", J Rehabil Res Dev, vol. 48, no. 6, pp. 643-659, 2011..

[4] C. Castellini, E. Gruppioni, A. Davalli, G. Sandini, "Fine detection of grasp force and posture by amputees via surface electromyography", J Physiol Paris, vol. 103, no. 3-5, pp. 255-262, Sep-Dec 2009.

[5] A.L. Alphonso, B.T. Monson, M.J. Zeher, R.S. Armiger, S.R. Weeks, J.M. Burck, C. Moran, R. Davoodie, G. Loeb, P.F. Pasquina, J.W. Tsao, "Use of a virtual integrated environment in prosthetic limb development and phantom limb pain", Stud Health Technol Inform vol. 181, pp. 305309, 2012.

[6] H. Flor, "Phantom-limb pain: characteristics, causes, and treatment", Lancet Neurol, vol. 1, no. 3, pp. 182-189, Jul 2002.

[7] D.A. Zlotolow, S.H. Kozin, "Advances in upper extremity prosthetics", Hand Clin, vol. 28, no. 4, pp. 587-593, Nov 2012.

[8] R.H. Chowdhury, M.B. Reaz, M.A. Ali, A.A. Bakar, K. Chellappan, T.G. Chang, "Surface electromyography signal processing and classification techniques", Sensors (Basel), vol. 13, no. 9, pp. 1243112466, Sep 2013.

[9] A. Phinyomark, P. Phukpattaranont, C. Limsakul, "Feature reduction and selection for EMG signal classification", Expert Systems with Applications, vol. 39, no. 8, pp. 7420-7431, 2012. 
[10] K Englehart, B Hudgins, PA Parker, "A wavelet-based continuous classification scheme for multifunction myoelectric control", IEEE Trans Biomed Eng, vol. 48, no. 3, pp. 302-311, Mar 2001.

[11] MA Oskoei, H Hu, "Support vector machine-based classification scheme for myoelectric control applied to upper limb", IEEE Trans Biomed Eng, vol. 55, no. 8, pp. 1956-1965, Aug. 2008.

[12] M. Khezri, M. Jahed, "An exploratory study to design a novel hand movement identification system", Comput Biol Med, vol. 39, no. 5, pp. 433-442, May 2009.

[13] K. Englehart, B. Hudgins, P.A. Parker, M. Stevenson, "Classification of the myoelectric signal using time-frequency based representations.", Medical engineering \& physics, vol. 21, no.6, pp. 431-438, 1999.
[14] C.M. Bishop, "Pattern Recognition and Machine Learning", Springer, Aug. 2006.

[15] J.M. Hahne, B. Graimann, K.R. Müller, "Spatial filtering for robust myoelectric control", IEEE Trans Biomed Eng, vol. 59, no.5, pp. 14361443, May 2012.

[16] F. Riillo, L.R. Quitadamo, F. Cavrini, E. Gruppioni, C. A. Pinto, N. C. Pastò, L. Sbernini, L. Albero, G. Saggio, "Supervised vs. unsupervised data preprocessing for EMG-based hand gesture recognition: a methodological study", unpublished. 